\title{
Etude comparée de la ventilation naturelle et automatique à l'aide de tuyaux perforés et du procédé classique de retournements des andains en compostage
}

\author{
Alaki-Issi Massimapatom SEMA ${ }^{1 *}$, Edem K. KOLEDZI ${ }^{1}$, Nitale M'Balikine KROU $^{2}$ et \\ Maglwa TCHA-THOM ${ }^{1}$ \\ ${ }^{1}$ Laboratoire de Gestion, Traitement et Valorisation des Déchets(GTVD), Université de Lomé, \\ BP 1515 Lomé, Togo. \\ ${ }^{2}$ Laboratoire d'Assainissement Sciences de l'Eau et Environnement (LASEE), Université de Kara, \\ B.P 404 Kara, Togo. \\ *Auteur correspondant, E-mail : alakisema@gmail.com; Tel : (00228) 91954088
}

\section{REMERCIEMENTS}

Ce travail a été financé par le Fonds SUEZ Environnement et SYCTOM en France à travers l'association GEVALOR-France et ENPRO-Togo.

Received: 27- 11- 2020
Published: 28- 02- 2021

\section{RESUME}

Sur la plateforme de compostage de l'ONG ENPRO, pour chaque tas de compost, cinq retournements sont nécessaires en vue d'apporter de l'air pour une bonne décomposition aérobie. Le retournement d'un seul tas de compost nécessite trois ouvriers pour une durée de trois heures. Donc, pour chaque fréquence de retournement, il faut mobiliser dix-sept ouvriers en une journée. Leur prise en charge pour les cinq retournements s'élève à 595000 FCFA. Face à ce coût élevé, la recherche d'alternatives de réduction du nombre d'ouvriers et du temps s'impose. La ventilation naturelle qui consiste à renouveler de l'air par des forces naturelles à travers des ouvertures, se présente comme la technique la plus optimiste. Ainsi, l'objectif de ce travail est d'optimiser la main d'œuvre et le temps accordé aux retournements. Cinq andains sont élaborés dont un standard et les quatre autres à ventilation naturelle. L'étude comparative des critères de maturité et de stabilité a montré des valeurs similaires pour les deux types de compost. Aussi, la ventilation naturelle a permis de réduire le nombre de retournements à deux et le nombre d'ouvriers à dix contre cinq et dix-sept pour le compostage standard puis le coût de prise en charge des ouvriers d'environ $25 \%$.

(C) 2021 International Formulae Group. All rights reserved.

Mots clés : Compostage, retournement des andains, optimisation du temps, ventilation naturelle.

\section{Comparative study of natural and automatic ventilation using perforated pipes and the classic method of turning windrows in composting}

\section{ABSTRACT}

On the composting platform of the NGO ENPRO, for each pile of compost, five turns are necessary in order to provide air for good aerobic decomposition. Turning a single compost heap requires three workers for three hours. So, for each turnaround frequency, seventeen workers must be mobilized in one day. Their cost for 
the five turnarounds amounts to FCFA 595,000. Faced with this high cost, the search for alternatives to reduce the number of workers and time is essential. Natural ventilation, which consists of renewing air by natural forces through openings, is presented as the most optimistic technique. Thus, the objective of this work is to optimize the manpower and the time allocated to turnarounds. Five windrows are produced, one of which is standard and the other four have natural ventilation. The comparative study of the maturity and stability criteria showed similar values for the two types of compost. Also, natural ventilation has reduced the number of turnovers to two and the number of workers to ten against five and seventeen for standard composting and the cost of supporting workers by around $25 \%$.

(C) 2021 International Formulae Group. All rights reserved.

Keywords: composting, windrow turning, time optimization, natural ventilation.

\section{INTRODUCTION}

L'évolution démographique et l'urbanisation croissante dans les Pays En Développement (PED) entraînent une production d'importantes quantités de déchets ménagers et industriels (Re-Source., 2015). Cette production croissante fait que certaines villes africaines comme Lomé rencontrent des difficultés dans la gestion des déchets (Kondoh et al., 2019). Relativement riches en matières organiques, ces déchets sont une opportunité s'ils ne sont pas mal gérés (incinérés, brulés ou transformés) pour les agriculteurs pour fertiliser ou améliorer la qualité des sols des parcelles cultivées. Pour cela, il est nécessaire d'améliorer les techniques de compostage de ces divers substrats organiques pour les sols cultivés, en favorisant la biodisponibilité des nutriments pour la plante cultivée. Le compostage de la fraction biodégradable qui constitue la fraction la plus importante des déchets, contribue à réduire l'émission du méthane qui est un Gaz à Effet de Serre responsable du réchauffement climatique. Plusieurs initiatives de compostage ont été menées dans les villes des PED car il constitue une voie intéressante pour le traitement de la fraction organique des déchets solides ménagers et assimilés (Tchegueni, 2011). Il existe une vaste gamme de technologies, méthodes et expériences en compostage dans ces PED. Cependant, l'enjeu est de pouvoir définir la technique la mieux appropriée à petite, moyenne et grande échelle en prenant en compte les aspects sociaux et économiques locaux. Sur la plateforme de l'Organisation Non Gouvernementale Ecosystème Naturel Propre (ONG ENPRO), l'aération par retournement présente trop d'inconvénients, parmi lesquels une perte de temps et une mobilisation d'un grand nombre d'ouvriers. La recherche d'alternatives pour optimiser le temps, l'implication des ouvriers avec une meilleure maîtrise des coûts à l'étape du retournement des andains s'impose.

$$
\text { Le compostage nécessite }
$$

d'importantes quantités d'oxygène, tout particulièrement lors $\mathrm{du}$ stade initial. L'aération est la source d'oxygène, et se trouve être ainsi un facteur indispensable pour le compostage aérobie. Quand l'approvisionnement en oxygène n'est pas suffisant, la croissance des micro-organismes aérobies se trouve limitée, ce qui ralentit la décomposition. Ainsi, une bonne aération est indispensable pour un compostage efficace (Turan et al., 2008). Selon la même étude, la consommation d'oxygène est généralement élevée dans la phase de fermentation et diminue au fur et à mesure que le processus de compostage évolue. Certains auteurs ont montré que la présence d'oxygène à un seuil minimal de l'ordre de $5 \%$ d'oxygène est indispensable au bon déroulement du compostage (Puyuelo et al., 2010). Cet oxygène est aussi consommé d'une part par les micro-organismes au cours de leur activité, et, d'autre part, par les réactions d'oxydation. 
Ce seuil de 5\% d'oxygène pourra être atteint si la qualité physique des matériaux (taille des particules et teneur en eau), la taille du tas et la ventilation est contrôlée et si le mélange est fréquemment retourné (Puyuelo et al., 2010). Plusieurs méthodes permettent d'apporter de l'air dans les andains notamment le retournement de l'andain, l'insufflation d'air ou système de ventilation mécanisé par utilisation de ventilateurs ou de tuyaux d'aération à l'intérieur de l'andain et de la ventilation naturelle (Zurbugg et al., 2005). Cependant, la ventilation se trouve à une place de choix dans le contexte où l'optimisation du temps, l'implication des ouvriers avec une meilleure maîtrise des coûts à l'étape du retournement des andains s'impose. En effet, la ventilation naturelle est une stratégie passive, sans moyen mécanique, de maintenir une aération intérieure confortable des andains. L'étude de la ventilation naturelle en compostage fait l'objet de recherche il y'a des années, notamment dans le cadre d'études relevant de l'aération des andains. Par ailleurs, la ventilation naturelle, si elle est bien maîtrisée, aide à réduire le nombre de retournements mais elle peut amener à une réduction des besoins d'ouvriers déployés pour cette tâche sur les plateformes de compostage. L'objectif de la présente étude était d'évaluer l'efficacité de la ventilation naturelle afin de réduire la main d'œuvre et le temps accordé aux retournements des andains.

\section{MATERIEL ET METHODES}

\section{Site d'étude}

Des essais de ventilation naturelle sont réalisés sur la plateforme de compostage de l'ONG ENPRO (Ecosystème Naturel Propre). La plateforme de compostage de l'ONG ENPRO de Longitude Est $01^{\circ} 10^{\prime} 54^{\prime \prime}$, et de latitude Nord $06^{\circ} 10^{\prime} 3$ " est localisée au nordouest de la ville de Lomé dans le $5^{\text {ième }}$ arrondissement du district d'Adidogomé.
Les déchets qui arrivent à la plateforme sont des déchets ménagers et assimilés de la ville de Lomé. Les déchets sont ensuite triés grâce à des tables de tri en vue d'obtenir les déchets compostables comme les putrescibles (restes de nourriture) et les papiers-cartons (Koledzi, 2011) tel qu'illustré sur la Photo 1.

ENPRO traite en moyenne 3989 \pm 277 tonnes de déchets par an et produit deux types de compost désigné sous le nom de «compost ENPRO »: le Standard (déchets ménagers seuls et assimilés) et l'Enrichi (Déchets ménagers et assimilés plus boue de vaches). La plateforme utilise 17 ouvriers pour ses activités de tris, mise en andain et retournement des tas jusqu'à maturation au bout de trois mois minimum. Elle utilise la technique classique de compostage à retournement manuelle avec 5 retournements ( $4^{\text {ième }}, 9^{\text {ième }}, 21^{\text {ième }}, 45^{\text {ième }}$ et $70^{\text {ième }}$ jour) au cours de la maturation avec un apport d'eau selon la nécessité (taux d'humidité des déchets). Le tri et la mise en andain d'un tas de 50 brouettes dure une journée maximum en saison humide et un peu moins en saison sèche où les déchets sont faciles à trier.

\section{Expérimentation de la technique de ventilation naturelle \\ Constitution des tas avec les systèmes d'aération}

Pour réaliser ces essais, les outils suivants ont été utilisés : une table de tri, les pelles, les pioches, les fourches, les grands seaux pour les refus, les petits sceaux pour les déchets dangereux, un trommel, les brouettes pour le transport du compost et des refus, la sulfureuse pour la mise en sac, les sacs de 50 $\mathrm{kg}$, les gants, les bottes, une balance pont bascule, un arrosoir de $11 \mathrm{~L}$, le thermomètre pour la mesure journalière de température, des bâches et de tuyaux PVC perforés. Le substrat qui sera utilisé est la fraction fermentescible des ordures ménagères.

Après le tri, les déchets compostables sont mis en andains sur lesquels sont 
positionnés les tuyaux perforés, la distance trou-trou étant de $40 \mathrm{~cm}$ (photo 2). Dans ce système, l'air circule dans les tuyaux et à travers l'andain en raison de l'effet de tirage créé par les gaz chauds qui s'élèvent hors de l'andain. Quatre (04) andains ont été formés avec le dispositif et un (01) comme standard (photo 3). Chaque andain est constitué de cinquante (50) brouettes de déchets correspondant à $1137 \pm 217 \mathrm{~kg}$ pour chaque andain. Tout le processus de mise en place des andains est décrit par la fiche technique (Figure 1).

Une fois l'andain mis en place, l'aération a été faite jusqu'au fond par un retournement manuel complet de toutes les couches du compost. En plus de l'aération, le compost a été aussi régulièrement (soit au cours des retournements ou lorsque l'on constate un en sèchement) humidifié pour moduler le taux d'humidité et garder ainsi le compost ni trop sec ni trop humide (Ngangué et al., 2012). Les quantités d'eau ajoutées au lancement est de $165 \mathrm{~L}$ pour le standard et $140 \pm 14 \mathrm{~L}$ pour les essais, au cours de la maturation $658 \mathrm{~L}$ pour le standard et $162 \pm 140$ L pour les essais. Le compost standard a été retourné cinq fois soit le $4^{\text {ième }}, 9^{\text {ième }}, 21^{\text {ième }}$, $45^{\text {ième }}$ et $70^{\text {ième }}$ jour alors que les andains avec tuyaux ont été retournés deux fois soit les $28^{\text {ième }}$ et $63^{\text {ième }}$ jours.

Pour le suivi, un échantillonnage s'est fait également chaque deux semaine au cours des trois mois comme suit: semaine 2, semaine 4 , semaine 6 , semaine 8 semaine 10 et semaine 12. Un seul protocole d'échantillonnage a été défini. Les échantillons ont été prélevés (le standard comme les andains ventilés) suivant le facteur profondeur et le facteur orientation (ou côté de l'andain) : prélèvements à 4 positions : Est, Nord, Ouest et Sud de l'andain.

\section{Paramètres de contrôle du processus de compostage}

Les échantillons de compost ont été prélevés suivant le facteur profondeur et le facteur orientation soit dans la longueur de l'andain, de chaque côté et à des profondeurs variables $(0,5 ; 1 ; 1,5 ; 2 \mathrm{~m})$ (Sandec, 2006 ; Unmar et al., 2008) en vue de la mesure des paramètres physico-chimiques comme la température le $\mathrm{pH}$, l'humidité, la matière organique et l'azote kjerdhal (NTK). En effet, la température des andains a été suivie tous les jours avec un thermomètre électronique lors du processus de dégradation (Koledzi, 2011).

Le $\mathrm{pH}$ a été mesuré sur une suspension aqueuse à l'aide d'un pH-mètre de marque Metrohm selon la méthode standard AFNOR NF ISO 10-390 (Belyaeva et al., 2009).

L'humidité $(\% \mathrm{H})$ a été déterminée selon la méthode normée Afnor NF U 44-171 d'octobre 1982 par la formule 1: $\% H=\frac{M_{0}-M_{1}}{M_{0}}$ (Formule 1);

Avec :

- $M_{0}$ : masse de l'échantillon brute (g)

- $M_{1}$ : masse de l'échantillon après passage à l'étuve (g)

- \%H : pourcentage d'humidité contenu dans l'échantillon

La teneur en matière organique totale (MO en $\%$ de MS) a été déterminée par la perte au feu selon la norme NF U 44-160 de novembre 1985 par la formule 2 :

$\%$ MOT $=\frac{M_{1}-M_{2}}{M_{1}}($ Formule 2$) ;$

Avec :

- $M_{1}$ : masse de l'échantillon après passage à l'étuve (g)

- $M_{2}$ : masse de l'échantillon après calcination (g)

- \%MOT : pourcentage de matière organique contenue dans l'échantillon

La teneur en azote total (NTK) des échantillons est déterminée en utilisant la méthode Kjeldhal telle que décrite par the Association of Official Analytical Chemists (AOAC). 


\section{Traitement statistique des données}

Les paramètres statistiques sont mis à contribution pour l'exploitation des résultats. Afin d'apprécier la distribution et les écarts sur les valeurs obtenues à partir des différentes méthodes utilisées, une moyenne $M$ et un écart type $\mathrm{E}$ sont calculés pour chaque résultat (Formules 3 et 4 ).
$M=\sum_{i=1}^{n} \frac{1}{n} \times Y_{i} \quad$ (Formule 3$) ;$

$E=\sqrt{\frac{\sum_{i=1}^{n}\left(Y_{i}-M\right)}{n-1}} \quad$ (Formule 4);

avec :

$\mathrm{M}=$ la moyenne arithmétique ; $\mathrm{Yi}=$ la mesure

$\mathrm{i} ; \mathrm{n}=$ le nombre de mesures.
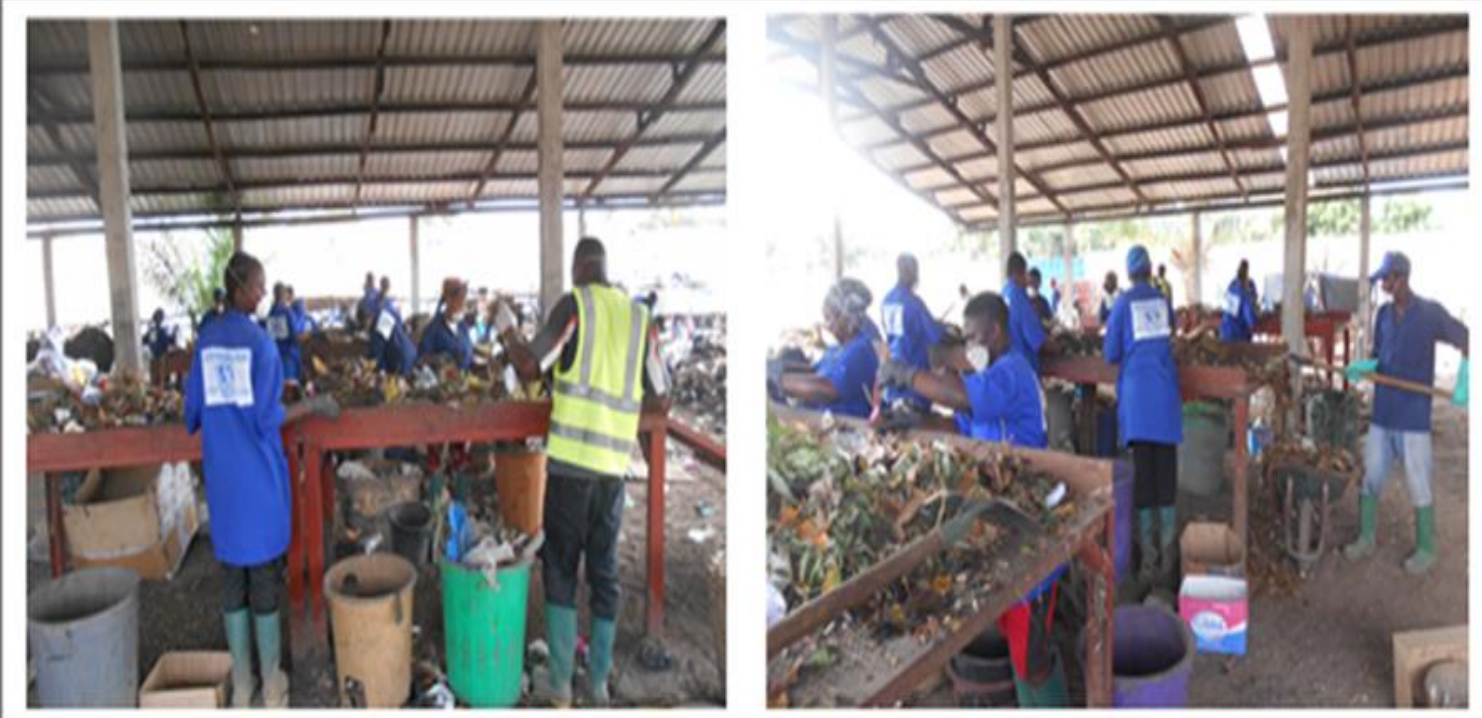

Photo 1 : Table de tri.

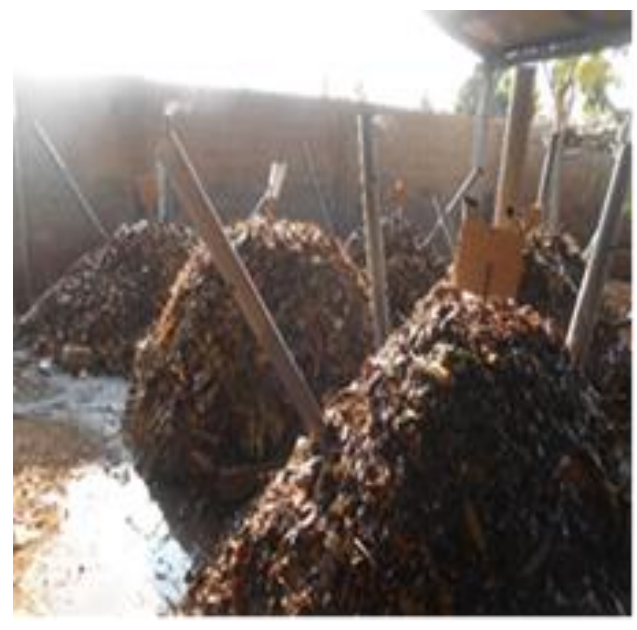

Photo 2 : Andain de ventilation naturelle.

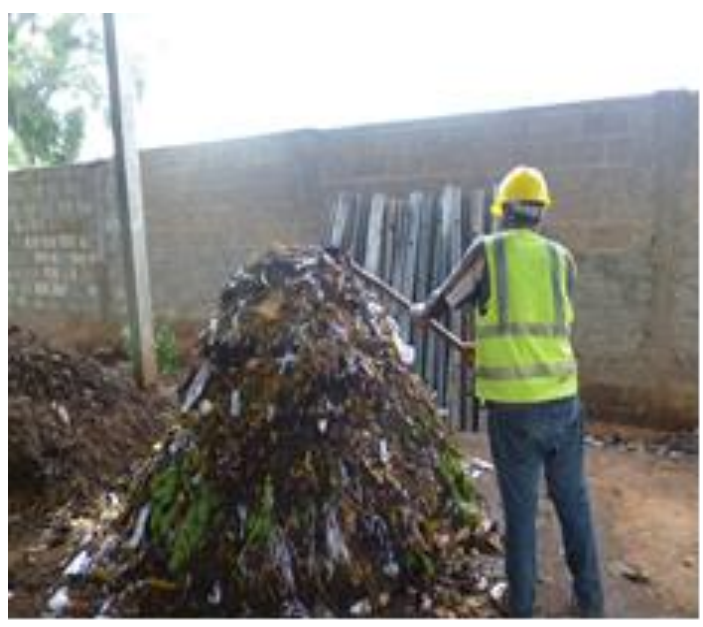

Photo 3 : L'andain Standard. 

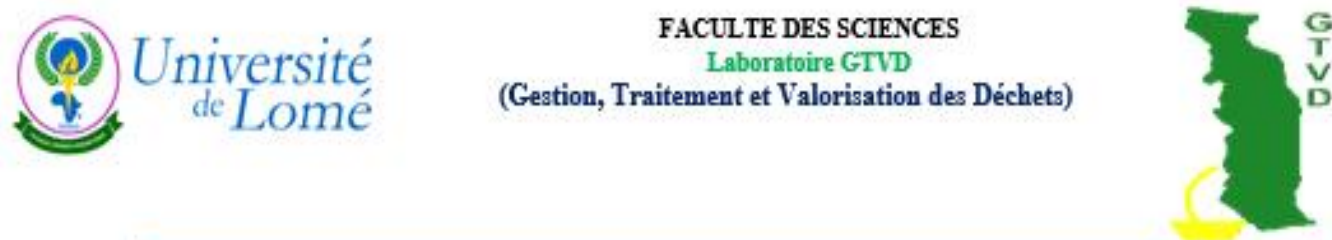

\section{FICHE TECHNIQUE DU PROCEDE DE COMPOSTAGE PAR VENTILLATION NATURELLE}
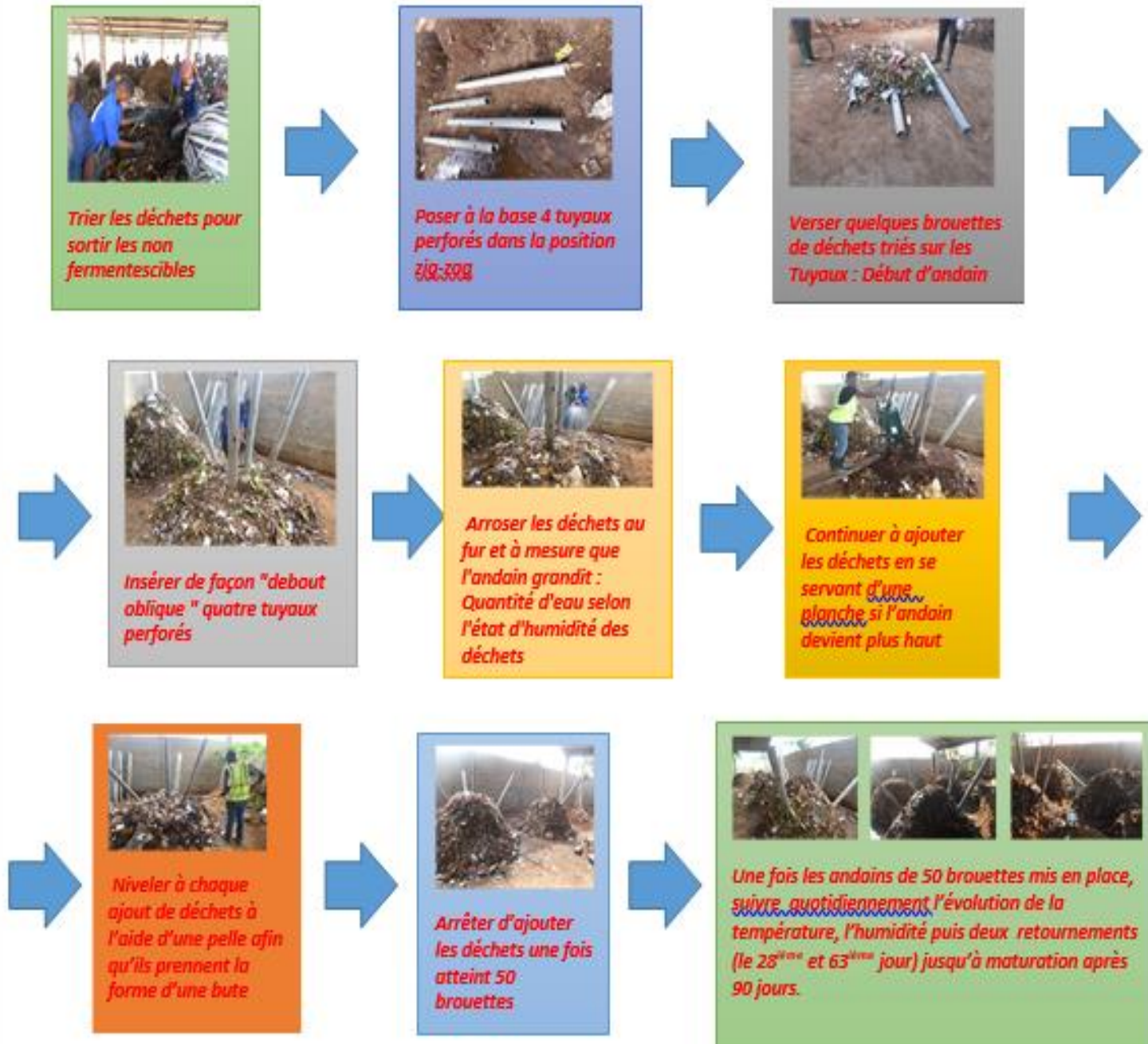

Une fois les andoins de 50 brovettes mis en place, suiver guetidienoement Yévolution de lo température, Yhumidité puis deux retournements (le $28^{\text {tat }}$ et $63^{\text {tina }}$ jour) jusqu'ò moturation après 90 jours.

Alaki-Issi M. SEMA (Dostargatidu

DrEdem K. KOLEDZI (MC)

Figure 1: Fiche technique du procédé de compostage par ventilation naturelle. 
RESULTATS

Paramètres physico-chimiques de suivi du processus de compostage

Le suivi de la température permet de s'assurer du niveau d'hygiénisation $\mathrm{du}$ compost des déchets. La Figure 2 présente l'évolution de la température lors $\mathrm{du}$ processus de compostage.

La Figure 3 présente la variation du $\mathrm{pH}$ (eau) des composts.
Les valeurs de l'évolution de la matière organique, du carbone organique total et d'azote total sont consignées dans le Tableau 1.

\section{Maturité des composts}

Les valeurs de $\mathrm{pH}$, les teneurs en azote total et de carbone organique total en fin du processus de compostage sont consignées dans le Tableau 2.

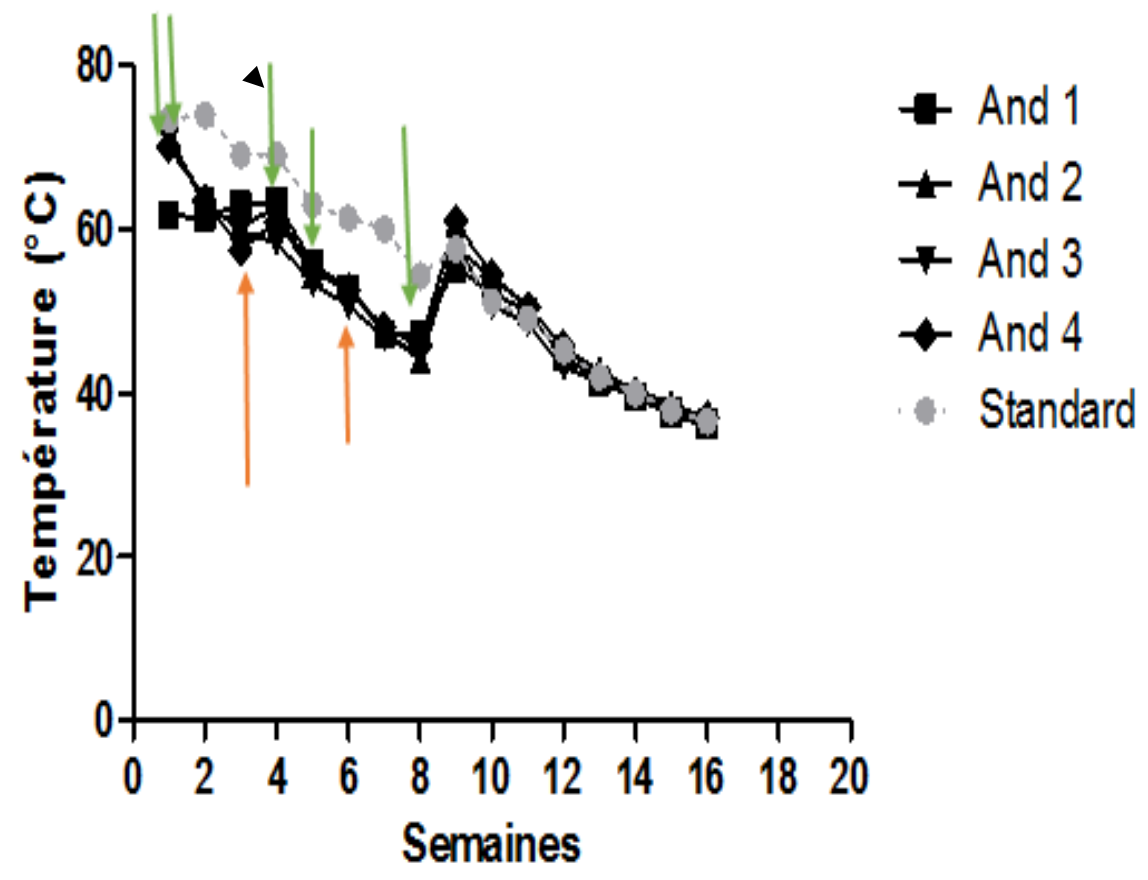

-Retournements du standard Retournements des essais

Figure 2 : Evolution hebdomadaire de la température. 


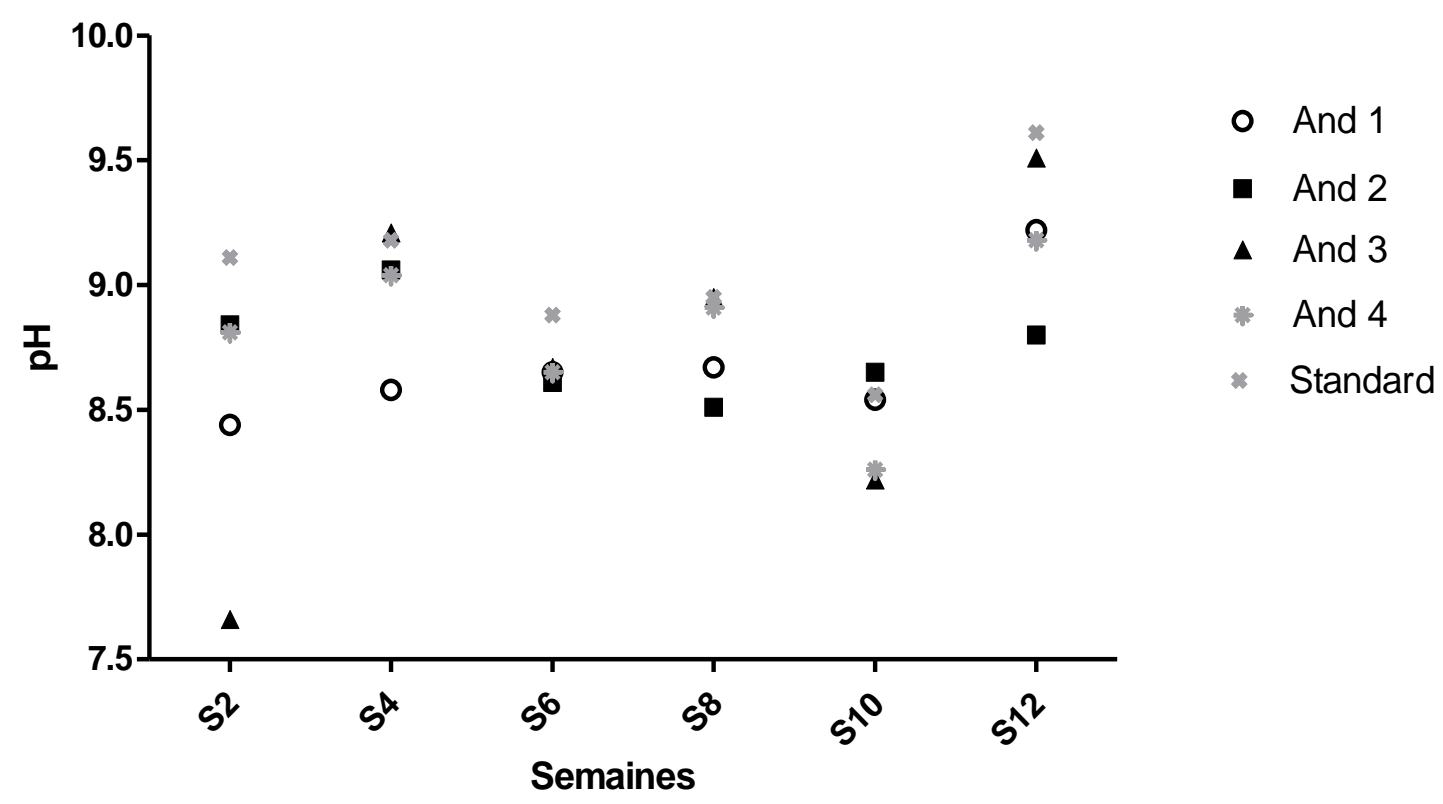

Figure 3 : Evolution du potentiel hydrogène.

Tableau 1 : Composition en matière organique et azote total.

\begin{tabular}{cccc}
\hline ANDAINS & \multicolumn{3}{c}{ Paramètres } \\
\hline & Carbone organique \% C & Matière Organique MO\% & Azote total \% N \\
\hline Standard (début) & 15,1 & 30,3 & 0,9 \\
\hline $\begin{array}{c}\text { Standard (maturité) } \\
\text { Essais (1, 2,3,4) }\end{array}$ & 11,5 & 23 & 0,70 \\
$\begin{array}{c}\text { (début) } \\
\text { (maturité) }\end{array}$ & 12,6 & 26 & 0,70 \\
2(maturité) & 11,0 & 22,1 & 0,5 \\
3(maturité) & 10,7 & 21,3 & 0,9 \\
4 (maturité) & 12,8 & 25,5 & 0,9 \\
& 8,2 & 16,3 & 1,1 \\
\hline
\end{tabular}


Tableau 2 : Le potentiel d'hydrogène, le carbone organique total, l'azote total et le rapport $\mathrm{C} / \mathrm{N}$ du compost.

\begin{tabular}{ccccccccc}
\hline Composts & Standard & And 1 & And 2 & And 3 & And 4 & ENPRO & $\begin{array}{l}\text { Castaldi et } \\
\text { al., 2008), } \\
\text { (Tchegueni et } \\
\text { al., 2012) }\end{array}$ & $\begin{array}{c}\text { (Toundou, } \\
\text { 2018) }\end{array}$ \\
& & & & & & & & \\
pH & $9,3 \pm 0,3$ & $8,9 \pm 0,6$ & $9,2 \pm 0,1$ & $9,3 \pm 0,2$ & $9,4 \pm 0,2$ & 8,9 & - & 8,5 \\
COT (\%) & $11 \pm 1$ & $11 \pm 2$ & $11 \pm 2$ & $13 \pm 1$ & $14 \pm 1$ & $12 \pm 2$ & $23-35$ & $12 \pm 4$ \\
NTK (\%) & $0,7 \pm 0,1$ & $0,53 \pm 0$, & $0,88 \pm 0,0$ & $0,9 \pm 0,1$ & $1,1 \pm 0,2$ & $0,90 \pm 0,0$ & $3,78-0,93$ & $0,92 \pm 0,10$ \\
C/N & 16 & 21 & 12 & 15 & 14 & 25 & - & 22,5 \\
\hline
\end{tabular}

And : andain

\section{DISCUSSION}

L'ensemble des essais de ventilation naturelle et le standard ont affiché des températures comprises entre $59{ }^{\circ} \mathrm{C}$ et $75{ }^{\circ} \mathrm{C}$ entre la première semaine et la quatrième semaine (Figure 2). Durant tout le processus de compostage, le standard est resté l'andain le plus chaud. La forte élévation de la température au sein de l'andain standard serait due à l'absence de la tuyauterie qui assure une ventilation continue des andains. Les températures de la $8^{\text {ième }}$ semaine sont restées stables autour de $42{ }^{\circ} \mathrm{C}$ (Figure 2) ; valeur supérieure à celle des essais de Toundou (2018). Les faibles valeurs de température enregistrées s'expliquent par une mauvaise circulation de l'oxygène nécessaire pour l'activité des micro-organismes (Toundou, 2018). Les andains 1, 2, 3 et 4 présentent une évolution similaire à celle du standard (Figure 2). Les pics de température observés les $4^{\text {ième }}$ et $9^{\text {ième }}$ semaines pour les andains ventilés naturellement correspondent aux deux retournements. Pour le compost standard, les températures sont montées après le deuxième et troisième retournement soit respectivement le $9^{\text {ième }}$ et $21^{\text {ième }}$ jour. Cependant, au quatrième retournement soit au $45^{\text {ième }}$ jour, on note une faible élévation de la température. Un cinquième retournement soit au $70^{\text {ième }}$ a été effectué afin de vérifier si la décomposition est terminée car un épuisement en oxygène pourrait aussi induire une baisse d'activité des micro-organismes entraînant une production de méthane, ce qui peut être corrigé par des apports complémentaires d'oxygène par retournement. Les températures ont presque évolué de la même manière jusqu'à maturation, bien que les essais étaient plus ventilés et moins retournés que le standard sauf que les températures des essais de ventilation sont restées un peu inférieurs à celles du standard. Les températures obtenues pour les essais de ventilation naturelle tout comme le compost standard sont suffisantes pour l'élimination des germes indésirables.

Les valeurs du $\mathrm{pH}$ sont comprises entre 9,6 et 10,4 à $10^{-2}$ près. Le $\mathrm{pH}$ des composts est légèrement alcalin $(9,6-10,4)$, ce qui est conforme aux valeurs de $\mathrm{pH}$ des composts matures (Koledzi, 2011 ; Yu et al., 2009 ; Sundberg et al., 2004 ; Lo et al., 2019). Ces valeurs offrent donc des conditions favorables à l'amélioration des propriétés biologiques et à la disponibilité des cations du sol. Le pH initialement de 6,5 a augmenté respectivement de 2 unités à la deuxième semaine et de 2,7 unités à maturité. Le pH de l'ensemble des andains sont devenus basiques dès la deuxième semaine $(8 \pm 0,6)$ et à maturité $(9,2 \pm 0,3)$ (Figure 3). Les valeurs de $\mathrm{pH}$ des quatre andains de ventilation naturelle 
sont similaires à celle du standard sauf l'andain 2 qui, à la semaine 2 avait un $\mathrm{pH}$ beaucoup plus faible. Les différentes valeurs obtenues durant la phase de maturation sont semblables à celles rapportées par la littérature 7 à 8,5 (Koledzi, 2011). La valeur de $\mathrm{pH}$ atteignant 9 est liée à une mobilisation importante d'ammonium $\mathrm{NH}_{4}{ }^{+}$à partir de l'azote organique des déchets et à la présence de cendre dans les ordures ménagères (Tchanate et al., 2017).

La minéralisation importante de la matière organique entraîne une diminution des teneurs en matières organiques au cours du compostage. Les composts se caractérisent donc par des teneurs en matière organique inférieures à celles des déchets bruts. La diminution relative de matière organique est très variable et dépend des conditions de compostage et de sa durée. Ces pertes peuvent atteindre 20 à $60 \%$ de la matière organique initiale (Krou et al., 2019). Les résultats de cette recherche confirment la littérature par un taux de matière organique élevé dans tous les andains au départ soit $30 \%$ pour le standard et $26 \%$ pour les essais. Ce taux diminue légèrement pour atteindre une valeur minimale moyenne de $23 \%$ pour le standard et $21,3 \pm 3,8 \%$ pour les essais de ventilation naturelle $(1,2,3,4)$ (Tableau 1). Les teneurs en matière organique par perte au feu en fin $\mathrm{du}$ processus sont similaires à celle du standard. Ces teneurs sont dans la fourchette rencontrée dans la littérature soit entre 20$40 \%$ pour un compost mûr (Koledzi, 2011). La dégradation de la matière organique entraîne une minéralisation importante du substrat au cours du compostage. Les composts se caractérisent donc par des teneurs en matière organique inférieures à celles des déchets bruts. Le taux de diminution de la matière organique du standard $(7,3 \%)$ est supérieur à celui des essais de ventilation naturelle $(4,7 \%)$, ce qui marque une dégradation plus importante du compost standard. Cela se justifie par le fait que les andains aérés à base des tuyaux perforés ayant subi moins de retournements se sèchent plus vite, ce qui ralentie la dégradation.
La teneur en carbone organique total diminue au cours du compostage comme la matière organique. La principale raison de cette diminution est l'utilisation par les microorganismes du milieu des substances organiques indispensables à leur métabolisme, conduisant à la minéralisation en dioxyde de carbone $\left(\mathrm{CO}_{2}\right)$. La présence possible de sites anaérobies dans la matrice peut entraîner des émissions de méthane $\left(\mathrm{CH}_{4}\right)$ liées à des métabolismes de type fermentaire (He et al., 2000) ; ces émissions représentant moins de 2\% du COT (Beck-Friis et al., 2003).

Ainsi, les taux de COT dans le compost sont de $3,6 \%$ pour le compost standard contre $1,9 \%$ pour les essais de ventilation (Tableau 1). Le faible taux pour les essais de ventilation par rapport à celui du compost standard serait lié à l'existence probable des sites anaérobies dans ces andains. Il faudra augmenter les tuyaux perforés pour une aération adéquate.

Le rapport $\mathrm{C} / \mathrm{N}$ moyen obtenu à partir du Tableau 3 est de 17 au début et 16 à maturité du standard puis 18 au début et 13,5 à maturité des essais de ventilation naturelle. Malgré une diminution en azote à maturité, le rapport $\mathrm{C} / \mathrm{N}$ du compost standard reste encore élevé. Par contre, celui des essais de ventilation naturelle est voisin de la valeur préconisée par la norme NFU 44 - 051 qui fixe ce rapport à 13 à maturité. Ceci serait lié à l'homogénéisation des déchets lors de la formation des andains. Le standard aurait plus reçu des déchets riches en carbone (par exemple la paille) plus que les autres tas. Ces valeurs sont dans l'intervalle indiqué dans la littérature pour un compost mûr soit un rapport $\mathrm{C} / \mathrm{N}$ compris entre $10-15$. Ce rapport a diminué au cours du compostage pour atteindre des valeurs comprises entre 8 et 25 , ce qui s'explique par le fait que les microorganismes consomment plus de carbone que d'azote. Les valeurs obtenues indiquent que les composts sont mûrs.

\section{Analyse de l'optimisation du temps de travail sur la plateforme}

L'étude comparative du coût d'investissement entre la ventilation naturelle 
et le compostage standard, a montré que seulement deux retournements sont nécessaires de la fermentation jusqu'à la maturation ; et pour chaque retournement, il faut dix (10) ouvriers pour la ventilation naturelle contre cinq (05) retournements et dix-sept (17) ouvriers pour le compostage standard. Le coût de mise en place de la ventilation naturelle (tuyaux et prise en charge de la main d'œuvre) s'élève à 450000 FCFA, contre 595000 FCFA pour le compostage standard. Ainsi, la ventilation naturelle permet de réduire le nombre d'ouvriers et aussi le coût de prise en charge des ouvriers d'environ $25 \%$. Ces résultats permettent de conclure que cette technique pourrait permettre de réduire le coût de prise en charge des ouvriers et le temps de retournement des andains durant le processus de compostage sur la Plateforme de l'ONG ENPRO.

\section{Conclusion}

Un ensemble d'analyses physicochimiques comme l'humidité, le $\mathrm{pH}$, la température, la matière organique, le carbone organique total et l'azote kjerdhal ont permis d'obtenir les indications qui définissent la qualité du compost produit. Les valeurs de $\mathrm{pH}$ en fin du processus, dans les composts sont basiques (8-9). Les teneurs en matière organique se situent en fin de maturation entre $26 \%$ et $30 \%$. Bien que retournés que 2 fois contre 5 fois pour le standard, les résultats des 4 composts ayant servi à l'essai de ventilation naturelle sont comparables à ceux du compost standard. En plus, le taux de diminution de la matière organique du standard $(7,3 \%)$ est supérieur à celui des essais $(4,7 \%)$ marquant une dégradation plus importante du standard ; le taux de diminution en COT du standard est de 3,6\% contre $1,9 \%$ pour les essais traduisant une émission faible des Gaz à Effet de Serre (GES) par la méthode à ventilation naturelle. En plus, le taux de compost pur dans les tas ventilés naturellement est largement supérieur $(44 \pm 8 \%)$ qu'au niveau du standard sauf l'andain 2 qui présente un taux de compost pur de $33 \%$ contre $36 \%$ pour le standard. En termes du coût d'investissement, les résultats obtenus montrent que la ventilation naturelle permet de réduire le nombre d'ouvriers et le coût de prise en charge des ouvriers d'environ $42 \%$. Cette technique peut être proposée pour une réduction de la main d'œuvre et du temps de retournement, mais elle nécessite un suivi régulier de la teneur en eau.

\section{CONFLIT D'INTERETS}

Les auteurs déclarent n'avoir aucun conflit d'intérêts.

\section{CONTRIBUTIONS DES AUTEURS}

A-IMS est l'auteur principal de ce travail. EKK a participé au montage du protocole et à l'analyse des résultats. NMK a participé aux premiers essais sur la plateforme de compostage et aux analyses chimiques au laboratoire. MT-T a participé à l'exploitation des résultats et à la discussion.

\section{REMERCIEMENTS}

Ce travail présenté dans ce document est réalisé dans le Laboratoire GTVD de l’Université de Lomé au Togo.

\section{REFERENCES}

Beck-Friis B, Jonsson S, Eklind $\mathrm{H}$, KirchmannY, Smars H. 2003. Composting of source-separated housold organics at different oxygen levels: gaining and undestanding of the emission dynamics. Compost Sci \& Utilizat., $\quad$ 11(1): $41-50$. DOI :10.1080/1065657X.2003.10702108 Belyaeva ON, Haynes RJ. 2009. Chemical, microbial and physical properties of manufactured soils produced by cocomposting municipal green waste with coal fly ash. Bioresource Technology, 100(21): $\quad 5203-5209 . \quad$ DOI: 10.1016/j.biortech.2009.05.032.

Castaldi P, Garau G, Melis P. 2008. Maturity of compost from muninicipal solid waste through the study enzyme activities and water-soluble fractions . Waste Manag., 28(3) : $\quad 534-540 . \quad$ DOI: 10.1016/j.wasman.2007.02.002.

Koledzi KE, Baba G, Feuillade G, Matejka G. 2011. Caractérisation physique des déchets solides urbains à Lomé au Togo, 
dans la perspective du compostage décentralisé dans les quartiers. Revue Francophone d'Ecologie Industrielle. Mars 2011. p 8-9.

Haug. 1993. The Practical Handbook of Compost Engineering. Boca Raton, Florida, 717.

He Y, Inamori $\mathrm{Y}$, Mizuochi M, Kong H, Iwami N, Sun T. 2000. Measurments of $\mathrm{N}_{2} \mathrm{O}$ and $\mathrm{CH}_{4}$ from aerated composting of food waste. Sci. Total Environ., 254(1): 65-74. DOI: 10.1016/s00489697(00)00439-3.

Kondoh E, Bodjona MB, Aziable E, Tchegueni S, Kili KA, Tchangbedji G. 2019. Etat des lieux de la gestion des déchets dans le Grand Lomé. Int. J. Biol. Chem. Sci., 13(4): 2200-2209. DOI :

https://dx.doi.org/10.4314/ijbcs.v13i4.25

Kolédzi KE. 2011. Valorisation des déchets solides urbains dans les quartiers de Lomé (Togo): Approche méthodologique pour une production durable de compost. Thèse de Doctorat Unique, Université de Lomé et Université de Limoges, p.224.

Krou NM, Baba B, Martín-Pascual J, Zamorano Toro M. 2019. Stabilization by co-composting dry drain sludge with fermentescible fractions of household garbage from the city of Sokodé. Int. J. Biol. Chem. Sci., 13(7): 3234-3246.

Lo M, Sonko M, Dieng D, Ndiaye S, Diop C, Seck A, Gueye A. 2019. Co-compostage de boues de vidange domestiques avec des déchets maraîchers et des déchets de poissons à Dakar (Sénégal). Int. J. Biol. Chem. Sci., 13(6) : 2914-2929.

Ngangué NM, Naquin P, Ngnikam E. 2012 .Compostage des déchets ménagers dans les pays en développement :Modalités de mise en place et de suivi d'installations décentralisées pérennes. Centre Francophone de Recherche Partenariale sur l'Assainissement, les Déchets et l'Environnement, 57.

Canet R, Pomares F. 1995. Changes in physical, chemical and physico-chemical parameters during the composting of municipal solid wastes in two plants in valencia. Bioresour Technol., 51(2-3): 259-264.

DOI : https://doi.org/10.1016/09608524(94)00132-K.

Puyuelo B, Gea T, Sanchez A. 2010. A new control strategy for the composting process based on the oxygen uptake rate. Chemical Engineering Journal, 165(1) : 161-169.

DOI: https://doi.org/10.1016/j.cej.2010.09.011

Re-Source P. 2015. Les techniques de compostage de déchets d'origine naturelle en Afrique et dans les caraïbes ; présentation synthétique de l'état des lieux et des retours d'expériences.

Sandec. 2006. Decentalised Composting for Cities of Low-and Middle-Income Countries. Waste Concern, House - 21 (Side B), Road - 7, Block - G, Banani Model Town, Dhaka-1213, Bangladesh. 61-62.

Sundberg C, Smars S, Jonsson H. 2004. Low $\mathrm{pH}$ as an inhibiting factor from mesophilic to thermophilic phase in composting. Bioresource Technology, 95: $145-150 . \quad$ DOI: http://dx.doi.org/10.1016/j.biortech.2004 .01 .016 .

Tchanate KN, Segbeaya KN, Koledzi KE, Baba G. 2017. Evaluation of the physicochemical and agronomic quality of the composts of urban waste of the towns of lome and Kara in Togo. European Journal of Scientific Research., 147(4): 469-474.

Tchegueni S, Kili AK, Bodjona M, Koriko M, Hafidi M, Baba G, Tchangbedji G. 2012. Effets des composts à base de déchets d'agrumes et du tourteau de karité sur la disponibilité du phosphore du sol: une étude en conditions contrôlées. Int. J. Biol. Chem. Sci., 6(3): 1381-1389. DOI: 10.4314/ijbcs.v6i3.39.

Tiquia SM, Tamn FY.1998. Composting pig mature in Hong Kong. Biocycle, 39(2) : 78-79.

Toundou Outéndé. 2018. Evaluation des caractéristiques chimiques et agronomiques de cinq composts de 
déchets et étude de leurs effets sur les propriétés chimiques du sol, la physiologie et le rendement du maïs (Zea mays L. Var. Ikenne) et de la tomate (Lycopersicum esculentum L. Var. Tropimech) sous deux régimes hydriques au Togo. Thèse de doctorat unique de l'Université de Lomé en cotutelle avec l'Université de Limoges ; Formation Doctorale: Biologie Végétale Appliquée; Spécialité: Physiologie Végétale.p214.

Unmar G, Mohee R. 2008. Assessing the effect of biodegradable and degradable plastics on the composting of green wastes and compost quality. Bioresource
Technology, 99(15): 6738-6744. DOI : https://doi.org/10.1016/j.biortech.2008.0 1.016.

Yu H, Huang GH. 2009. Effects of sodium acetate as a $\mathrm{pH}$ control amendment on the Composting of food waste. Bioresource. Technology, 100 (6): 20052011.

DOI: 10.1016/j.biortech.2008.10.007.

Zurbugg C, Drescher SS. 2005. Decentralized composting in Bangladesh, a win-win situation for all stakeholsers. Resources Conservation and Recyling, 43(3) : 281292.

DOI: 